\title{
Processos de incubação artificial associados à aplicação de diferentes métodos reprodutivos em matrizes semipesadas
}

\author{
Incubation artificial processes associated with the application of different reproductive \\ methods in matrices semi heavy
}

\author{
RUFINO, João Paulo Ferreira ${ }^{1}$; CRUZ, Frank George Guimarães ${ }^{1}$; MACHADO, \\ Noédson de Jesus Beltrão ${ }^{2}$; BRASIL, Ronner Joaquim Mendonça ${ }^{2}$; PEREIRA, Pedro \\ Augusto Marques ${ }^{1}$; FARIAS, Eliena Guimarães ${ }^{1}$
}

\footnotetext{
${ }^{1}$ Universidade Federal do Amazonas, Faculdade de Ciências Agrárias, Setor de Avicultura, Manaus, Amazonas, Brasil.

${ }^{2}$ Universidade Federal do Amazonas, Instituto de Ciências Sociais, Educação e Zootecnia, Parintins, Amazonas, Brasil.

*Endereço para correspondência: frankcruz@ufam.edu.br
}

RESUMO

O objetivo deste trabalho foi estudar diferentes métodos reprodutivos em aves de duas linhagens semipesadas e avaliar seus efeitos sobre os processos de incubação artificial dos ovos e do rendimento de carcaça dos pintainhos. O delineamento experimental foi inteiramente casualisado em esquema fatorial $(2 \times 2)$ com os fatores: métodos reprodutivos (inseminação artificial e monta natural) e duas linhagens semipesadas (Plymouth Rock Barrada e Rhode Island Red). Foram utilizadas 26 matrizes (23 fêmeas e 3 machos) para cada fator que originaram 62 ovos cada, totalizando 248 ovos, sendo cada ovo uma repetição. Os ovos foram pesados antes e após o período de incubação para determinação da perda de peso e realização de análise comparativa entre o peso do ovo e o peso do pinto. Durante o período de incubação foi avaliado o desempenho reprodutivo das matrizes, e após o nascimento, foram abatidos 8 pintos de cada fator para análise do rendimento de carcaça dos mesmos. Não foram encontradas diferenças significativas $(\mathrm{P}>0,05)$ entre $\mathrm{O}$ desempenho reprodutivo e a relação entre $\mathrm{O}$ peso do pinto e o peso do ovo. O peso do saco vitelino e peso do fígado foram afetados $(\mathrm{P}<0,05)$ pelos tratamentos tendo a linhagem Plymouth Rock Barrada apresentado melhores resultados.

Palavras-chave: desempenho reprodutivo, inseminação artificial, monta natural, rendimento de carcaça

\section{SUMMARY}

The objective of this study was different reproductive methods in birds from two semiheavy lineages and evaluate their effects on the processes of artificial incubation of eggs and carcass yield of chicks. The experimental design was completely randomized factorial $(2 \times 2)$ with factors: reproductive methods (artificial insemination and natural breeding) and two semi-heavy lineages (Plymouth Barred Rock and Rhode Island Red). Were used 26 matrices (23 females and 3 males) for each factor that led to 62 eggs each, totaling 248 eggs, each egg being a repeat. The eggs were weighed before and after the incubation period to determine weight loss and performing comparative analysis between the egg weight and the weight of chick. During the incubation period was evaluated the reproductive performance of the matrices, and after birth, 8 chicks each factor were killed for analysis of carcass thereof. No significant differences $(\mathrm{P}>0.05$ ) were found between reproductive performance and the relationship between chick weight and egg weight. The yolk sac and liver weights were affected $(\mathrm{P}<0.05)$ by treatment with a Plymouth Rock Barred strain showed the best results.

Keywords: artificial insemination, natural breeding, productive performance, yield of carcass 


\section{INTRODUÇÃOO}

No contexto da indústria avícola moderna, a fertilidade das matrizes é um dos pontos específicos a serem analisados, pois a mesma reflete $\mathrm{o}$ máximo retorno econômico a partir do número e da qualidade dos pintos produzidos por ave que serão comercializados. A eficiência reprodutiva das matrizes é determinada por diversos fatores, dentre os quais se destacam as instalações, os programas de iluminação, o manejo, a nutrição e a herança genética, estes que influenciam diretamente a capacidade da matriz de expressar seu potencial.

Para alcançar este máximo desempenho reprodutivo é necessário profundo conhecimento dos fatores que influenciam a maturação sexual das matrizes, a fertilidade, os processos relacionados à formação do embrião, a formação do ovo como estrutura e por fim a oviposição por parte das aves. Além destes, vários são os fatores ambientais que atuam sobre a fisiologia reprodutiva das aves, tais como temperatura, clima, disponibilidade de alimento e interações sociais (LESKA \& DUSZA, 2007).

Inserida neste contexto, a biotecnologia é uma da ferramenta importante no processo reprodutivo das aves, visando o desenvolvimento de técnicas ligadas principalmente à inseminação artificial e à criopreservação de sêmen (RUTZ et al., 2007), sendo que a conservação da variabilidade genética em espécies de animais domésticos é um desafio para a produção sustentável de recursos alimentares para os humanos e para o manejo animal (BLESBOIS et al., 2007) como contexto generalizado.

$\mathrm{O}$ incubatório na cadeia produtiva de aves atua como uma unidade de transformação biotecnológica, onde o ovo fecundado com o embrião em pleno desenvolvimento transforma-se no pintainho, $\mathrm{o}$ produto final a ser disponibilizado no mercado. Tendo em vista a importância de técnicas ligadas à reprodução e melhoramento genético de aves e a busca incessante da indústria de reprodução avícola por linhagens possuam melhores índices de produção e qualidade dos pintos, o presente trabalho foi realizado com o objetivo de aplicar diferentes métodos reprodutivos em aves selecionadas de duas linhagens semipesadas específicas e avaliar seus efeitos sobre os rendimentos obtidos nos processos de incubação artificial dos ovos e no rendimento de carcaça dos pintainhos nascidos.

\section{MATERIAL E MÉTODOS}

O experimento foi realizado no incubatório do Setor de Avicultura do Departamento de Produção Animal e Vegetal (DPAV) da Faculdade de Ciências Agrárias (FCA) da Universidade Federal do Amazonas (UFAM), localizado no Setor Sul do Campus Universitário, Manaus Amazonas.

Foram utilizados 248 ovos incubáveis de matrizes semipesadas com 32 semanas de idade e com peso médio de $2,64 \pm 0,3 \mathrm{~kg}$ para os machos e $2,14 \pm 0,2 \mathrm{~kg}$ para as fêmeas. Utilizou-se 104 matrizes semipesadas (92 fêmeas e 12 machos), sendo 52 aves correspondentes a linhagem Plymouth Rock Barrada e 52 aves à linhagem Rhode Island Red.

O delineamento experimental utilizado foi o inteiramente casualisado em esquema fatorial $(2 \times 2)$ com os fatores correspondentes: métodos reprodutivos (inseminação artificial e monta natural) 
e linhagens semipesadas (Plymouth Rock Barrada e Rhode Island Red).

Os tratamentos foram constituídos da seguinte forma: A - 62 ovos oriundos de aves da linhagem Plymouth Rock Barrada submetidas à monta natural, B - 62 ovos oriundos de aves da linhagem Plymouth Rock Barrada submetidas à inseminação artificial, C - 62 ovos oriundos de aves da linhagem Rhode Island Red submetidas à monta natural e D - 62 ovos oriundos de aves da linhagem Rhode Island Red submetidas à inseminação artificial.

Cada tratamento possuiu 26 aves (23 fêmeas e 3 machos) que originaram 62 ovos incubáveis cada durante sete dias de coleta, sendo que os ovos corresponderam a repetição gerada por cada tratamento e as aves à unidade experimental constituinte do mesmo.

Os ovos à medida que foram coletados, limpos e desinfetados até completarem os sete dias de coleta foram transportados para sala de armazenamento com temperatura de $18^{\circ} \mathrm{C}$ e umidade relativa de $74 \%$, respectivamente, permanecendo nesta até o momento da incubação, preservando assim a integridade do embrião e desconsiderando demais fatores de influencia externa que poderiam vir a interferir nos rendimentos de incubação.

As aves selecionadas para monta natural foram alojadas em boxes de $9 \mathrm{~m}^{2} \mathrm{com}$ cama de maravalha sendo um box para cada linhagem estudada, enquanto as aves utilizadas para inseminação artificial foram alojadas em 12 gaiolas (6 para cada tratamento) medindo $1,00 \times 0,40 \times 0,45 \mathrm{~m}$ cada e submetidas a inseminação artificial 15 dias antes do início do experimento.

As aves utilizadas na inseminação artificial foram alojadas em fileiras de gaiolas separadas por linhagem e os galos em gaiolas individuais. Posteriormente, os galos foram submetidos ao processo de coleta de sêmen por meio de massagem abdominal no dorso e movimentos nas laterais da cloaca sendo o sêmen armazenado em pequenos frascos descartáveis tipo eppendorf, para logo em seguida o mesmo procedimento ser aplicado na reversão da cloaca das matrizes e o sêmen ser introduzido no oviduto das mesmas através da utilização de uma pipeta de volume fixo de $0,025 \mathrm{~mL}$, obedecendo à metodologia proposta por Etches (1996). Todas as matrizes foram submetidas a idênticas condições de manejo e as rações isonutritivas à base de milho e farelo de soja foram formuladas obedecendo as recomendações de Rostagno et al. (2011).

Após resfriamento, os ovos foram pesados e passaram por um préaquecimento de seis horas na sala de incubação antes de serem incubados. Foi utilizada incubadora modelo PETERSIME 168 regulada para manter constante a temperatura do bulbo seco em $37,8^{\circ} \mathrm{C}$ e umidade relativa de $60 \%$. Com 19 dias de incubação, foi realizada a ovoscopia e os ovos claros foram retirados para identificação dos inférteis ou dos embriões mortos e a pesagem para mensuração da perda de peso dos ovos durante a transferência. Os ovos foram transferidos para bandejas de eclosão, as quais foram dispostas ao acaso em um nascedouro modelo PETERSIME 168 com termostato programado para manter a temperatura do bulbo seco em $36,6^{\circ} \mathrm{C}$ e umidade relativa de $65 \%$. A retirada dos pintos do nascedouro ocorreu com 504 horas (21 dias) sendo registrados os pintos nascidos para avaliação dos rendimentos de incubação e do peso do pinto.

Posteriormente ao nascimento, foram abatidos oito pintos de cada tratamento através de deslocamento cervical para 
Rev. Bras. Saúde Prod. Anim., Salvador, v.15, n.3, p.765-773 jul./set., 2014 http://www.rbspa.ufba.br ISSN 15199940

realização do rendimento de carcaça, onde foram avaliados os seguintes parâmetros: comprimento do sistema digestório, peso do coração, peso do pró-ventrículo + moela, peso do fígado e peso do saco vitelino.

Os dados coletados foram submetidos à análise de variância e para comparação das médias estimadas foi aplicado o teste de Tukey ao nível de $5 \%$ de probabilidade utilizando o programa computacional Statistical Analysis System - SAS (2008).

\section{RESULTADOS E DISCUSSÃO}

De acordo com os resultados encontrados não foram observadas diferenças significativas $(\mathrm{P}>0,05)$ para peso do ovo, perda de peso do ovo durante o período de incubação e peso do pinto ao nascer entre métodos reprodutivos, entre linhagens e na interação entre os fatores, Tabela 1.

Tabela 1. Peso do ovo ao ser incubado, percentual de perda de peso dos ovos durante o período de incubação e peso do pinto ao nascer

\begin{tabular}{lccc}
\hline \multirow{2}{*}{ Fatores } & \multicolumn{3}{l}{ Variáveis } \\
\cline { 2 - 4 } & Peso do Ovo & Perda de Peso & Peso do Pinto \\
\hline Método Reprodutivo & & & \\
\hline Monta Natural & 56,20 & 13,50 & 38,55 \\
Inseminação & 57,35 & 14,03 & 38,77 \\
\hline Linhagem & & & 38,31 \\
\hline Plymouth Rock Barrada & 56,27 & 13,35 & 39,00 \\
Rhode Island Red & 57,28 & 14,18 & 0,54 \\
\hline Efeito & & P Valor & 0,07 \\
\hline Método Reprodutivo & 0,31 & 0,58 & 0,27 \\
Linhagem & 0,37 & 0,39 & 0,26 \\
Interação & 0,72 & 0,52 & \\
\hline Coeficiente de Variação (\%) & 1,25 & 1,38 & \\
\hline
\end{tabular}

O peso do pinto ao nascer apresentou comportamento similar ao peso do ovo de origem independente da linhagem ou do método reprodutivo avaliado, constatando-se também que os ovos de maior peso originaram pintos com maior peso. Rocha et al. (2008) afirmaram que o rendimento de incubação, o peso e a qualidade do pinto dependem de vários fatores que incluem, entre outros, a idade da matriz que, por sua vez, influencia o peso do ovo, causando assim uma interação entre estes fatores.

A perda de peso do ovo durante o período de incubação está amplamente associada às condições de temperatura e umidade presentes na máquina de incubação e a qualidade da casca dos ovos, onde a água atravessa os poros da casca movendo-se sempre do ponto mais úmido, que normalmente é o interior do ovo, para o ponto mais seco, o ambiente. Já a umidade em volta dos ovos férteis deve ser controlada para assegurar desenvolvimento adequado dos embriões (DECUYPERE et al., 2001). Os ovos da linhagem Plymouth Rock Barrada apresentaram menor perda de peso em relação aos ovos da linhagem Rhode Island Red, porém, esta perda mantem-se proporcional ao 
peso do ovo e com valores aproximados. Meir \& Ar (2008), ao avaliarem o rendimento de incubação de ovos de matrizes leves durante o primeiro ciclo de produção e após a muda forçada estabeleceram que para preservar uma incubação dentro dos parâmetros normais de perda de peso do ovo deve-se trabalhar com ajustes graduais na umidade relativa da incubadora a fim de otimizar a produção.

Os resultados de desempenho reprodutivo das matrizes não apresentaram diferenças significativas entre métodos reprodutivos, linhagens e na interação entre os fatores $(P>0,05)$, conforme descrito na Tabela 2. Na eclosão, os resultados corroboram com Fiúza et al. (2006) que encontraram rendimentos de eclosão entre 83 e $86 \%$ incubando ovos férteis em diferentes condições de ambiente. Diversos são os fatores que podem interferir na eclosão durante o processo de incubação dos ovos, sendo a grande maioria proveniente de fatores ambientais, entre esses, a temperatura e a umidade relativa do ar são as principais variáveis que podem interferir (SANTANA et al., 2013), também afetando a eclodibilidade e a qualidade do pintinho, pois estes são influenciadas pelo tempo, pela temperatura e pela umidade de estocagem. Devido a escassez de referencias e informação científica relacionada a rendimentos de incubação de matrizes semipesadas, torna-se uma necessidade e até mesmo comum a utilização de dados oriundos de matrizes pesadas (MICHALSKY et al., 2005) que sirvam de parâmetros comparativos nestes estudos.

Tabela 2. Valores médios de taxa de fertilidade, taxa de eclodibilidade, taxa de eclosão e taxa de refugos de linhagens semipesadas

\begin{tabular}{lcccc}
\hline \multirow{2}{*}{ Fatores } & \multicolumn{4}{c}{ Variáveis } \\
\cline { 2 - 5 } & Fertilidade (\%) & Eclodibilidade (\%) & Eclosão (\%) & Refugos (\%) \\
\hline Método Reprodutivo & \multicolumn{5}{c}{} \\
\hline Monta Natural & 92,04 & 89,35 & 82,42 & 6,255 \\
Inseminação & 95,98 & 88,26 & 84,69 & 3,725 \\
\hline Linhagem & \multicolumn{5}{c}{80,75} & 2,815 \\
\hline Plymouth Rock Barrada & 91,96 & 87,59 & 7,165 \\
Rhode Island Red & 96,06 & 90,02 & 86,36 & 0,07 \\
\hline Efeito & \multicolumn{4}{c}{ P Valor } \\
\hline Método Reprodutivo & 0,06 & 0,08 & 0,67 & 0,10 \\
Linhagem & 0,09 & 0,06 & 0,31 & 0,11 \\
Interação & 0,06 & 0,07 & 0,22 & 9,63 \\
\hline Coeficiente de Variação (\%) & 3,57 & 8,26 & 7,72 & \\
\hline
\end{tabular}

As aves submetidas à inseminação artificial não apresentaram diferença significa $(\mathrm{P}>0,05)$ na taxa de fertilidade em relação às aves submetidas à monta natural e nem entre os fatores analisados e na interação entre estes. A proposta primordial da inseminação artificial aplicada a aves preza que todas as aves recebam o sêmen, enquanto as aves submetidas à monta natural nem sempre possuem a comprovação que todas realizaram a cópula. $\mathrm{E}$ mesmo que todas as aves tenham recebido o sêmen do macho através da inseminação, não é certeza absoluta que todos os ovos irão ter pleno desenvolvimento embrionário, 
pois o processo de incubação torna-o dependente de fatores físicos $\mathrm{e}$ fisiológicos (TONA et al., 2003).

Outrora, talvez a maior problemática encontrada na inseminação artificial não esteja associada aos índices reprodutivos das matrizes ou ao rendimento de carcaça dos pintos nascidos ou até mesmo a eficiência do método, mas sim a disponibilidade de mão de obra tecnificada para a realização de um programa de inseminação aplicado de forma contínua. Lavor \& Câmara (2012) discutem de forma sucinta o intenso investimento da comunidade científica em pesquisas relacionadas a biotecnologias aplicadas a reprodução de aves e sua relação com as indústrias e os centros de reprodução avícolas, pontuando que a utilização de inseminação artificial, assim como outras biotecnologias, depende $\mathrm{d}$ a disponibilidade de mão-de-obra especializada e interfere significativamente no custo de produção.

Quanto à mortalidade, os pintos oriundos da linhagem Plymouth Rock Barrada não apresentaram diferenças significativas $\quad(\mathrm{P}>0,05) \quad$ quando comparados com os pintos da linhagem Rhode Island Red tanto entre linhagens quanto entre métodos reprodutivos e na interação dos fatores. Wishart et al. (2005) estudando a linhagem Rhode Island Red em comparação com outras linhagens afirmam que este fato não pode ser atribuído a uma só causa específica, pois, em geral a mortalidade embrionária durante $\mathrm{o}$ período de incubação aumenta com a idade da matriz (mais relacionada com queda de fertilidade), com o período de estocagem do ovo (mortalidade embrionária no início e final da incubação) e com a falta de viragem do ovo na incubação e na estocagem (principalmente de aves mais velhas).
Os resultados apresentados pela linhagem Plymouth Rock Barrada (exceto na refugagem), segundo afirma Etches (1996), podem depender de vários fatores como a dose inseminante, idade do galo, da galinha, método de coleta de sêmen, intervalo entre coleta e inseminação, técnica de inseminação e qualquer outro fator que possa afetar a capacidade de fertilização e consequente desenvolvimento do embrião, posteriormente.

O rendimento de carcaça dos pintos apresentou diferença significativa $(\mathrm{P}<0,05)$ para peso do saco vitelino (entre linhagens), peso do fígado (entre métodos reprodutivos), Tabela 3 , e na interação entre os fatores para ambas as variáveis, Tabela 4 . O peso absoluto do saco vitelino de aves inseminadas artificialmente apresentou melhor resultado, corroborando com Lourens et al. (2006), que afirmam que a superioridade dos pesos absoluto e relativo do saco vitelino deve-se pela maior quantidade de nutrientes presente na gema dos ovos mais pesados.

Os resultados para saco vitelino apresentaram comportamento discrepante em relação ao peso do pinto, discordando dos resultados encontrados por Sklan et al. (2003). Pintos de maior peso podem apresentar carcaças bem desenvolvidas e sacos vitelinos reduzidos, isso se deve ao seu maior desenvolvimento durante $\mathrm{o}$ período de eclosão, ou carcaças menos desenvolvidas e sacos vitelinos maiores, o que os fornece uma sobrevivência mais longa antes de iniciar seu metabolismo alimentar exógeno. $\mathrm{O}$ peso do fígado também apresentou efeito significativo $(\mathrm{P}<0,05)$ entre linhagens $\mathrm{e}$ na interação dos fatores, tendo a linhagem Rhode Island Red, apresentado resultados superiores para inseminação artificial independente da linhagem avaliada. 
Rev. Bras. Saúde Prod. Anim., Salvador, v.15, n.3, p.765-773 jul./set., 2014 http://www.rbspa.ufba.br ISSN 15199940

Tabela 3. Valores médios para rendimento de carcaça de pintos de 1 dia de linhagens submetidos a diferentes métodos reprodutivos

\begin{tabular}{|c|c|c|c|c|c|}
\hline \multirow[b]{2}{*}{ Fatores } & \multicolumn{4}{|c|}{ Variáveis } & \multirow[b]{2}{*}{$\begin{array}{l}\text { Saco vit. } \\
\text { (g) }\end{array}$} \\
\hline & $\begin{array}{l}\text { Comp. Sist. Dig. } \\
(\mathrm{cm})\end{array}$ & $\begin{array}{c}\text { Coração } \\
(\mathrm{g})\end{array}$ & $\begin{array}{c}\text { Pró-ventrículo + } \\
\text { moela }(\%)\end{array}$ & $\begin{array}{l}\text { Fígado } \\
(\mathrm{g})\end{array}$ & \\
\hline \multicolumn{6}{|l|}{ Método Reprodutivo } \\
\hline Monta Natural & $44,86^{\mathrm{a}}$ & 0,36 & $2,57^{\mathrm{a}}$ & 0,99 & $4,45^{b}$ \\
\hline Inseminação & $41,15^{\mathrm{b}}$ & 0,37 & $2,25^{\mathrm{b}}$ & 1,10 & $5,78^{\mathrm{a}}$ \\
\hline \multicolumn{6}{|l|}{ Linhagem } \\
\hline $\begin{array}{l}\text { Plymouth Rock } \\
\text { Barrada }\end{array}$ & $45,11^{\mathrm{a}}$ & $0,34^{\mathrm{b}}$ & 2,50 & $0,91^{\mathrm{b}}$ & 4,98 \\
\hline Rhode Island Red & $40,90^{\mathrm{b}}$ & $0,40^{\mathrm{a}}$ & 2,32 & $1,18^{\mathrm{a}}$ & 5,25 \\
\hline Efeito & & & Valor & & \\
\hline Método Reprodutivo & $0,01 *$ & 0,65 & $0,01^{*}$ & 0,15 & $0,01 *$ \\
\hline Linhagem & $0,01 *$ & $0,03 *$ & 0,14 & $0,01 *$ & 0,36 \\
\hline Interação & 0,99 & 0,30 & 0,49 & $0,03 *$ & $0,04 *$ \\
\hline $\begin{array}{l}\text { Coeficiente de } \\
\text { Variação }(\%)\end{array}$ & 4,25 & 14,50 & 13,45 & 19,64 & 16,60 \\
\hline
\end{tabular}

Tabela 4. Valores médios para interação entre os fatores (métodos reprodutivos $\mathrm{x}$ linhagens) sobre peso do fígado e peso do saco vitelino

\begin{tabular}{|c|c|c|}
\hline \multirow{2}{*}{ Métodos Reprodutivos } & \multicolumn{2}{|c|}{ Linhagens Semipesadas } \\
\hline & Rhode Island Red & Plymouth Rock Barrada \\
\hline & \multicolumn{2}{|c|}{ Peso do fígado $(\mathrm{g})$} \\
\hline Monta Natural & $0,94^{\mathrm{Aa}}$ & $1,05^{\mathrm{Ab}}$ \\
\hline Inseminação artificial & $0,89^{\mathrm{Ba}}$ & $1,31^{\mathrm{Aa}}$ \\
\hline \multirow[t]{2}{*}{$\mathrm{CV}(\%)$} & \multicolumn{2}{|c|}{19,64} \\
\hline & \multicolumn{2}{|c|}{ Peso do saco vitelino $(\mathrm{g})$} \\
\hline Monta Natural & $4,00^{\mathrm{Bb}}$ & $4,90^{\mathrm{Aa}}$ \\
\hline Inseminação artificial & $5,95^{\mathrm{Aa}}$ & $5,60^{\mathrm{Aa}}$ \\
\hline $\mathrm{CV}(\%)$ & \multicolumn{2}{|c|}{16,60} \\
\hline
\end{tabular}

O peso do coração foi afetado significativamente $\quad(\mathrm{P}<0,05)$ pelos tratamentos entre linhagens, discordando de Wineland e Christensen (2003) que trabalhando com níveis de umidade relativa na incubação de ovos de matrizes pesadas sobre a relação peso do coração/peso do pinto não observaram diferença significativa em nenhum dos fatores analisados. $\mathrm{O}$ peso do pró-ventrículo + moela e o comprimento do sistema digestório foram influenciados significativamente $(\mathrm{P}>0,05)$ pelos tratamentos entre métodos reprodutivos, apresentando comportamento proporcional entre si, onde pintos oriundos de matrizes submetidas à monta natural apresentaram maior comprimento digestivo e consequente maior peso do pró-ventrículo + moela, podendo essa relação ser atribuída à correlação entre as funções destes para a nutrição e manutenção dos pintos durante seu pós-nascimento e 
Rev. Bras. Saúde Prod. Anim., Salvador, v.15, n.3, p.765-773 jul./set., 2014 http://www.rbspa.ufba.br ISSN 15199940

desenvolvimento. A inseminação artificial aplicada a linhagens semipesadas apresenta-se como alternativa viável e mais eficiente que a monta natural, principalmente para a linhagem Rhode Island Red, quando associada aos processos de incubação artificial relacionados aos índices produtivos das matrizes e rendimento de carcaça da progênie. Porém, apresenta a desvantagem da exigência de mão de obra especializada e necessária para a realização de um programa contínuo e eficiente aplicado a matrizes de pleno rendimento reprodutivo em escala de produção industrial de pintos.

\section{REFERÊNCIAS}

BLESBOIS, E.; SEIGNEURIN, F.; GRASSEAU, I.; LIMOUSIN, C.; BESNARD, J.; GOURICHON, D.; COQUERELLE, G.; RAULT, P.; TIXER-BOIXARD, M. Semen cryopreservation for ex situ management of genetic diversity in chicken: creation of the French Avian Cryobank. Poultry Science, v.86, p.555-564, 2007.

DECUYPERE, E.; TONA, K.; BRUGGEMAN, V.; BAMELIS, F. The day-old chick: A crucial hinge between breeders and broilers. World's Poultry Science Journal, v.57, p.127-138, 2001.

\section{ETCHES, R.J. Reproduction in} poultry. Wallingford, UK: CAB International, 1996.

FIÚZA, M. A.; LARA, L.J.C.; AGUILAR, C.A.L.; RIBEIRO; B.R.C.; BAIÃO, N.C. Efeitos das condições ambientais no período entre a postura e o armazenamento de ovos de matrizes pesadas sobre o rendimento de incubação. Arquivo Brasileiro de Medicina Veterinária e Zootecnia, v.58, p.408-413, 2006.

LAVOR, C.T.B.; CÂMARA, S.R. Biotecnologia do sêmen e inseminação artificial em aves. Ciência Animal, v.22, n.1, p.66-81, 2012.

LESKA, A.; DUSZA, L. Seasonal changes in the hypothalamo-pituitarygonadal axis in birds. Reproductive Biology, v.7, n.2, p.99-126, 2007.

LOURENS, A.; MOLENAAR, R.; VAN DEN BRAND, H.; HEETKAMP, M.J.; MEIJERHOF, R.; KEMP, B. Effect of egg size on heat production and the transition of energy from egg to hatchling. World's Poultry Science Journal, v.85, p.770-776, 2006.

MEIR, M.; AR, A. Changes in eggshell conductance, water loss and hatchability of layer hens with flock age and moulting. British Poultry Science, v.49, n.6, p.677-684, 2008.

MICHALSKY, V.B.; CANÇADO, S.V.; LARA, L.J.C.; BAIÃO,N.C.; SANTOS,G.C. Influência da umidade na incubação e idade da matriz leve sobre a eclosão e parâmetros de ovos e pintos. Revista Brasileira de Ciência Avícola, v.7, p.13, 2005. Supl.

ROCHA, J. S. R.; LARA, L. J. C.; BAIÃO, N. C.; CANÇADO, S. V.; BAIÃO, L. E. C., SILVA, T. R. Efeito da classificação dos ovos sobre o rendimento de incubação e os pesos do pinto e do saco vitelino. Arquivo Brasileiro de Medicina Veterinária e Zootecnia, v.60, n.4, p.979-986, 2008.

ROSTAGNO, H.S.; ALBINO, L.F.T.; DONZELE, J.L.; GOMES, P.C.; OLIVEIRA, R.F.; LOPES, D.C.; FERREIRA, A.S.; BARRETO, S.L.T. 
Tabelas brasileiras para aves e suínos: composição de alimentos e exigências nutricionais. Viçosa:

Universidade Federal de Viçosa, 2011.

RUTZ, F.; ANCIUTI, M. A.; XAVIER, E. G.; ROLL, V. F. B.; ROSSI, P. Avanços na fisiologia e desempenho reprodutivo de aves domésticas.

Revista Brasileira de Reprodução Animal, v.31, n.3, p.307-317, 2007.

SANTANA, M. H. M.; GIVISIEZ, P. E. N.; JÚNIOR, J. P. F.; SANTOS, E. G. dos. Avaliação de protótipos de incubadoras sobre os parâmetros embrionários de ovos férteis caipiras.

Revista de Ciências Agrárias, v.36, n.2, p, 2013.

STATISTICAL ANALYSIS SYSTEM. SAS/STAT Software. Version 9.2. Cary: SAS Institute Inc., 2008.

SKLAN, D.; HEIFETZ, S.; HALEVY, O. Heavier chicks at hatch improves marketing body weight by enhancing skeletal muscle growth. World's

Poultry Science Journal, v.82, p.17781786, 2003.
TONA, K.; MALHEIROS, R.D.;

BAMELIS, F.; CAREGHI, C.;

MORAES, V.M; ONAGBESAN, O.; DECUVPERE, E.; BRUGGEMAN, V. Effects of storange time on incubating egg gás pressure, thyroid hormones, and corticosterone levels in embryos and on their hatching parameters. World's Poultry Science Journal, v.82, p.840845, 2003.

WINELAND, M. J.; CHRISTENSEN, V. L. Effect of egg moisture loss upon the embryo and growing chick.

International Hatchery Practice, v.17, p.17, 2003.

WISHART, G. J., YOUNG, M., STAINES, H. J. Weekly monitoring of broiler flock mating efficiency by sperm transfer into eggs. British Poultry Science, v.45, p.400-403, 2005.

Data de recebimento: 27/12/2013

Data de aprovação: 19/08/2014 